\title{
MEMS technology for production of different micro- and nanodevices
}

\author{
A.M. Kudanovich, N.I. Mukhurov, L.R. Paleuskaya, Y.V. Mironchyk \\ Laboratory of Micro- Electronics, Mechanics and Sensorics, Institute of Physics of National Academy of \\ Sciences of Belarus \\ 220090, Institute of Physics of National Academy of Sciences of Belarus, \\ Logoyskiy trakt, 22, Minsk, Belarus \\ e-mail: kudanovich@inel.bas-net.by
}

To obtain of different microdevices apply techniques such as LIGA, surface and bulk micromashinning of silicon. However, they either can not provide a wide range of microstructures, or extremely expensive because they require special equipment and materials. It therefore remains an urgent problem and develop a universal low-cost technology, which will generate different kinds of microdevices on wide range of sizes that can work at high thermal and mechanical loads.

The proposed technology based on the processes of electrochemical oxidation of aluminum and bulk etching of the thus obtained oxide. The initial thickness of the aluminum plate is selected commensurate with the thickness of the insulating products to reduce time and expense of materials during etching of unreacted aluminum.

Grown aluminum oxide layer, depending on the used electrolyte and anodizing conditions can have a thickness from tenths to several hundred micrometers. Due to the availability of selective etchants for aluminum and their oxide, the grown layers of anodic alumina can be easily obtained in the free form of aluminum. In the initial state anodic alumina is in amorphous phase. When heated above $800{ }^{\circ} \mathrm{C}$ oxide transforms into a polycrystalline phase, which is characterized by a number of structural phases with specific physical-chemical and mechanical properties. Satisfactory electrical and mechanical properties create preconditions for the widespread use of anodic $\mathrm{Al}_{2} \mathrm{O}_{3}$ and its structural phases in creating elements of micromechanics and microsensorics and microelectromehanics.

Amorphous aluminum oxide does not undergo significant structural changes to $500^{\circ} \mathrm{C}$. At higher temperatures, the oxide crystalline phases are detected. A number of polymorphic transformations ends sustainable $\alpha$-phase (corundum) formed rapidly when heated to $1200^{\circ} \mathrm{C}$. At lower temperatures produced a mixture of metastable $\theta, \delta, \gamma$ and other phases, which are more or less stable at temperatures below the beginning of their formation. At a temperature of $850-950^{\circ} \mathrm{C}$ preferential amount of amorphous aluminum oxide becomes $\mathrm{Y}-\mathrm{Al}_{2} \mathrm{O}_{3}$ [1]. It must be emphasized that the such crystalline phase in terms of requirements for the dielectric elements of micromechanics, has a certain elasticity and mechanical strength, including those for relatively extended elements when the thickness from a few micrometers and above. Besides the unstable $y$-phase does not undergo any visible changes when exposed for several thousand hours at a temperature of $100-150^{\circ} \mathrm{C}$ lower than the pre-emptive formation of $\mathrm{y}$-phase. This characteristic is almost essential when using dielectric elements of micromechanics work at high temperatures $\left(500 \leq \mathrm{T} \leq 800^{\circ} \mathrm{C}\right)$.

Films of anodic aluminum oxide (AAO) have a unique self-organized morphology formed by homogeneous, and nanosized pores that extend the entire length of the film (Figure 1). It is a porous structure of anodic aluminum oxide is the determining factor for its high-precision etching. Density of transport pores, which are delivered to the metal-oxide oxygen or oxygen-containing complexes of $10^{10}$ $10^{12} \mathrm{~cm}^{-2}$. Pore diameters, depending on the electrolyte used and the mode of oxidation is $10-100$ nanometers. Due to the porosity the selective etchtant penetrates into the pores and the oxide is etched throughout the thickness. Theoretically, the lateral etching of oxide is comparable with the wall thickness of the oxide cell (it is approximately equal to the diameter of the pores).

Figure 2 shows the elements of the aluminum oxide thickness of 300 micrometers, obtained by etching. It is noticeable that all the elements of sharp vertical edges, practically no lateral etching.

The basic principle of obtaining metal and metal-ceramic (composite) microelements, based on aluminum oxide technology is electroplating, which assumes galvanic deposition of metal in forming matrixes. For the manufacture of metal micronutrients formed ceramic matrix (Figure 3 ) in the manner described above. Base ceramic matrix is aluminum. Then in the matrix by galvanic process were deposited nickel or other metal. After complete filling of the matrix metal oxide is completely or partially etched in a neutral to nickel (or other metal) etchant. The obtained metal microstructure of high-quality fine-grained layers of nickel (Figure 4) repeat the relief of the matrix with the same sharpness of the edge and have high mechanical strength. Elements of a thick and can be obtained from any metal deposited electrolytically. Upon otaining of composite microstructures adhesion of metal to ceramics is satisfactory, 
composite material under load is not destroyed. Perhaps filling the forming matrix and polymerizing plastics and other materials.
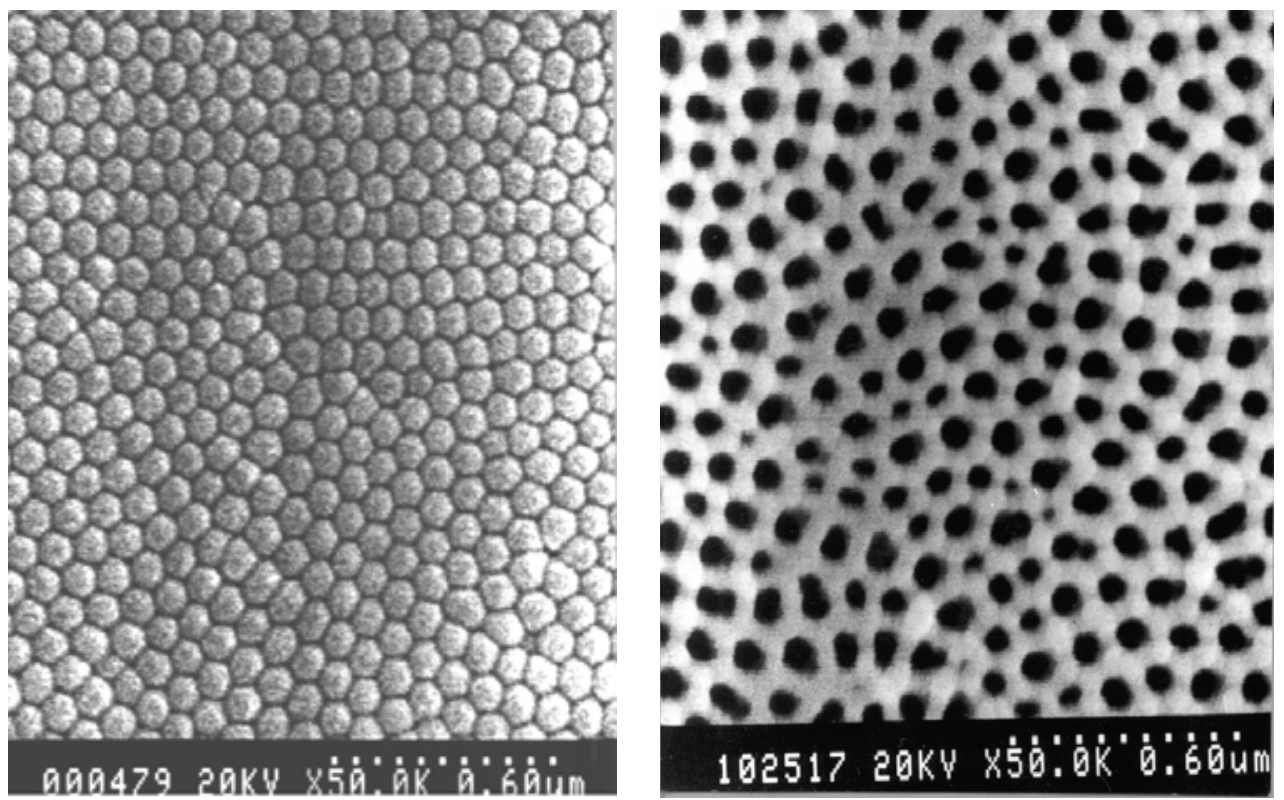

Figure 1. Morphology of $\mathrm{Al}_{2} \mathrm{O}_{3}$

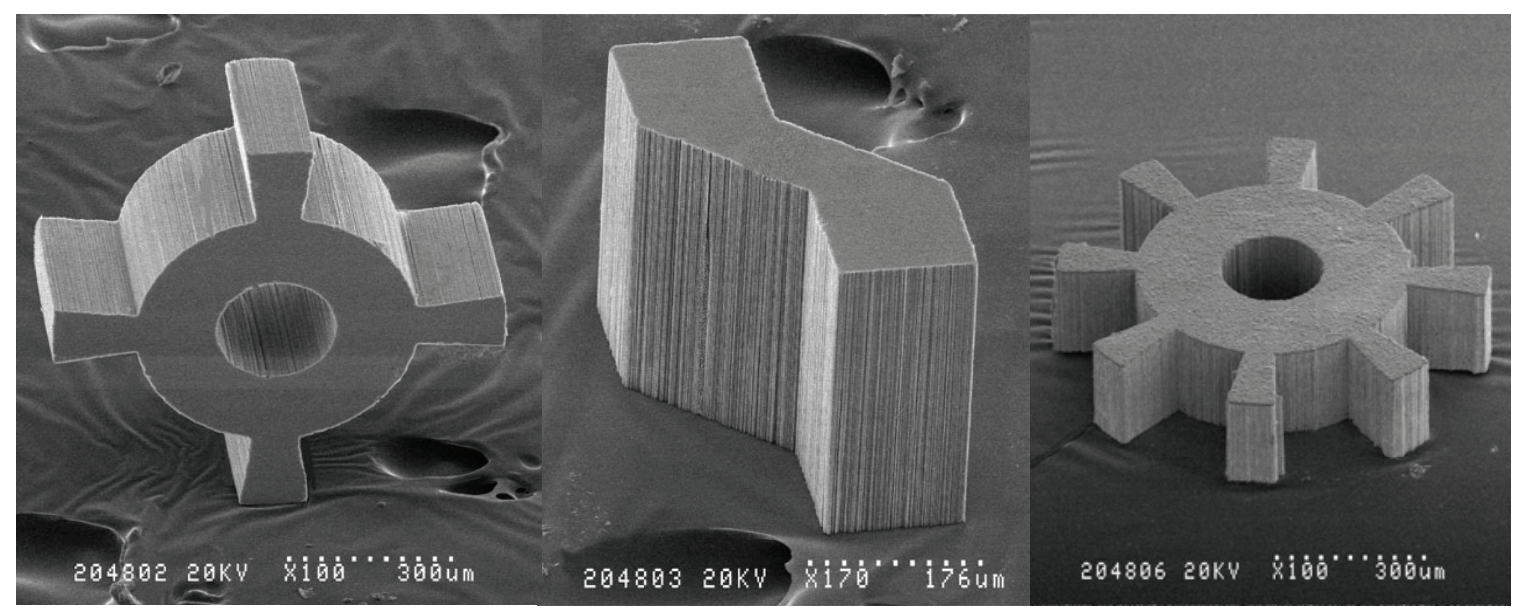

Figure 2. Microelements from $\mathrm{Al}_{2} \mathrm{O}_{3}$

Also developed a fabricated method for multilevel 2-D and 3-dimensional structures such as ceramics and metals, as well as from their combination. This approach is realized by again anodized aluminum substrate, which has formed the first level of $\mathrm{Al}_{2} \mathrm{O}_{3}$. The second level is then formed as the first. This approach has limited application and by exposing the photoresist on nonplanar substrate. Another way is to form $\mathrm{Al}_{2} \mathrm{O}_{3}$ on both sides of an aluminum foil. Samples can then be subjected to micromachining on both surfaces, followed by partial etching of the aluminum substrate. Note that the process can be realized in a wide range of thicknesses of $\mathrm{Al}_{2} \mathrm{O}_{3}$, thus providing unique opportunities for the design, for hybrid devices, sensors, various actuators.

Opportunities of the technology demonstrated by many microcomponents and microdevices prototypes [2, 3]. Created microheater with low energy consumption for a wide range of IR sources and gas microsensors. Temperature about $1150^{\circ} \mathrm{C}$ can be achieved with energy consumption of $10 \mathrm{~mW}$ at $100^{\circ} \mathrm{C}$. Nanoporous morphology of $\mathrm{Al}_{2} \mathrm{O}_{3}$ also opens up great opportunities for production baseline microsensors, which takes a lot of detailed surface with controlled porosity. These sensors show high sensitivity, chemical and mechanical stability, fast response. Figure 5 shows the elements of the vibration 
sensor, where the increase of inertial mass deposited metal into the hole, and it is firmly within the bore even with heavy vibration.

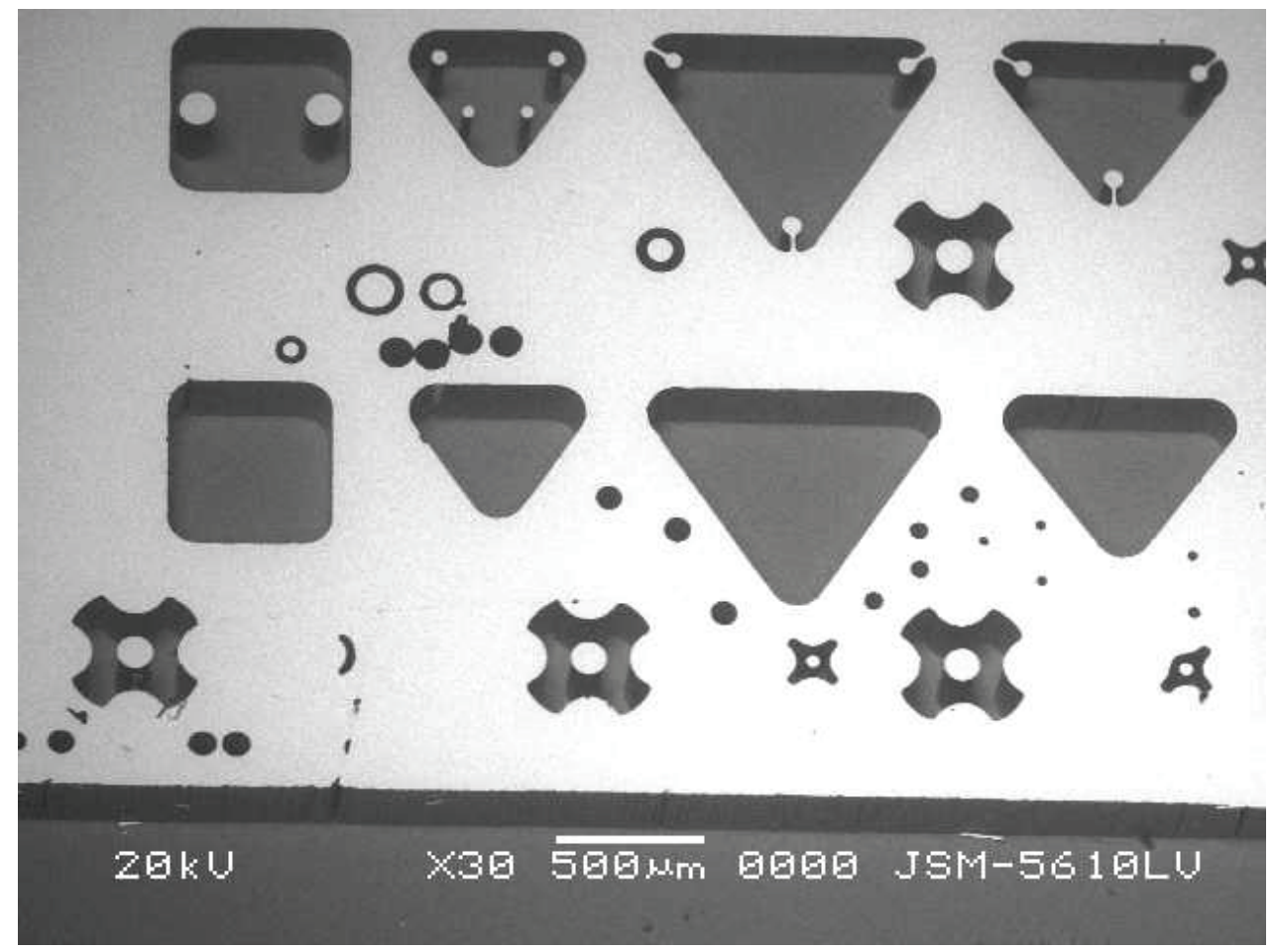

Figure 3. Matrix for galvanic deposition of metals from $\mathrm{Al}_{2} \mathrm{O}_{3}$

Other applications of $\mathrm{Al}_{2} \mathrm{O}_{3}$ technology include the creation of miniaturized energy sources, gas microturbines, membrane reactors for catalytic conversion of fuel, electrodes and membranes for fuel cells, micro-analysis systems components, filters, membranes, aperture, nozzle, etc. Described the material and its processing method makes possible the creation of hybrid ceramic MEMS with unique opportunities for both bulk and surface micromachining. Produced ceramic MEMS mechanically, chemically and thermally stable and can be used in high temperature conditions, the levels of radiation and corrosive conditions.

Further development of technologies based on alumina is the transition from micron to nanoscale. Previously indicated that the alumina has a unique self-organizing nanoporous structure. And if you create microelements pores were mostly used as a transport way for the enchants, the pores in the nanodevices will be used as the matrix in which it will be possible to obtain different compounds and thus to create unique nanoscale devices. At present synthesis technology developed in the channels has a wide range of materials (metals, semiconductors and magnetic materials). Depending on the type of material being implemented there are several main areas.

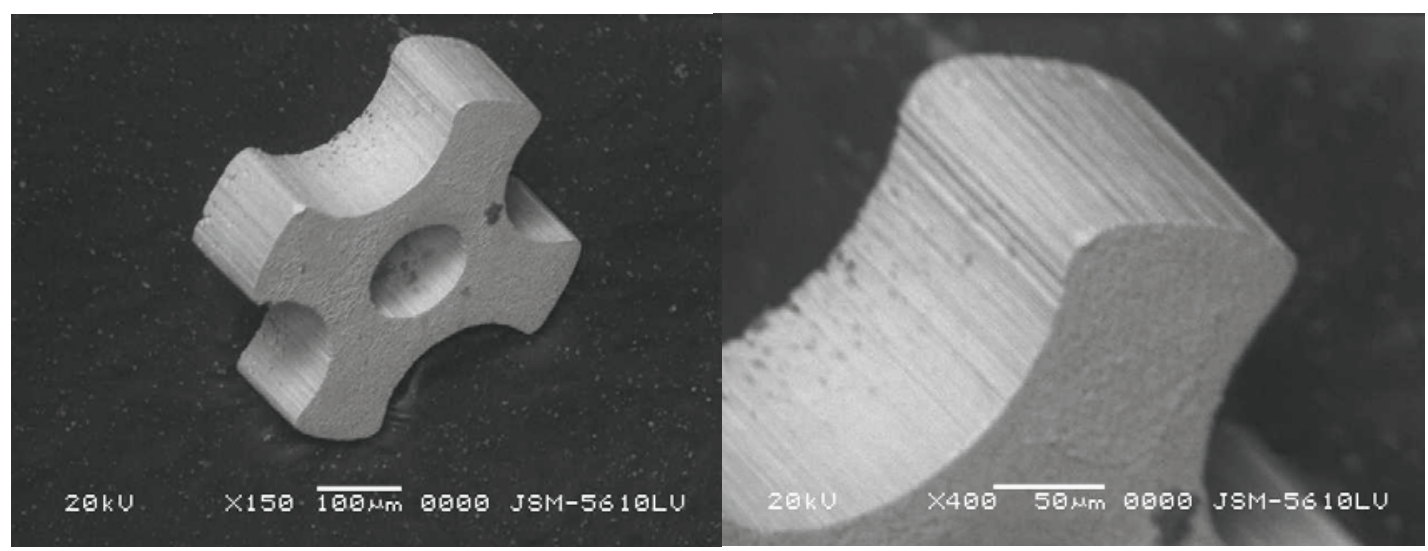

Figure 4. Metallic microgear, produced by galvanic deposition on forming matrix 


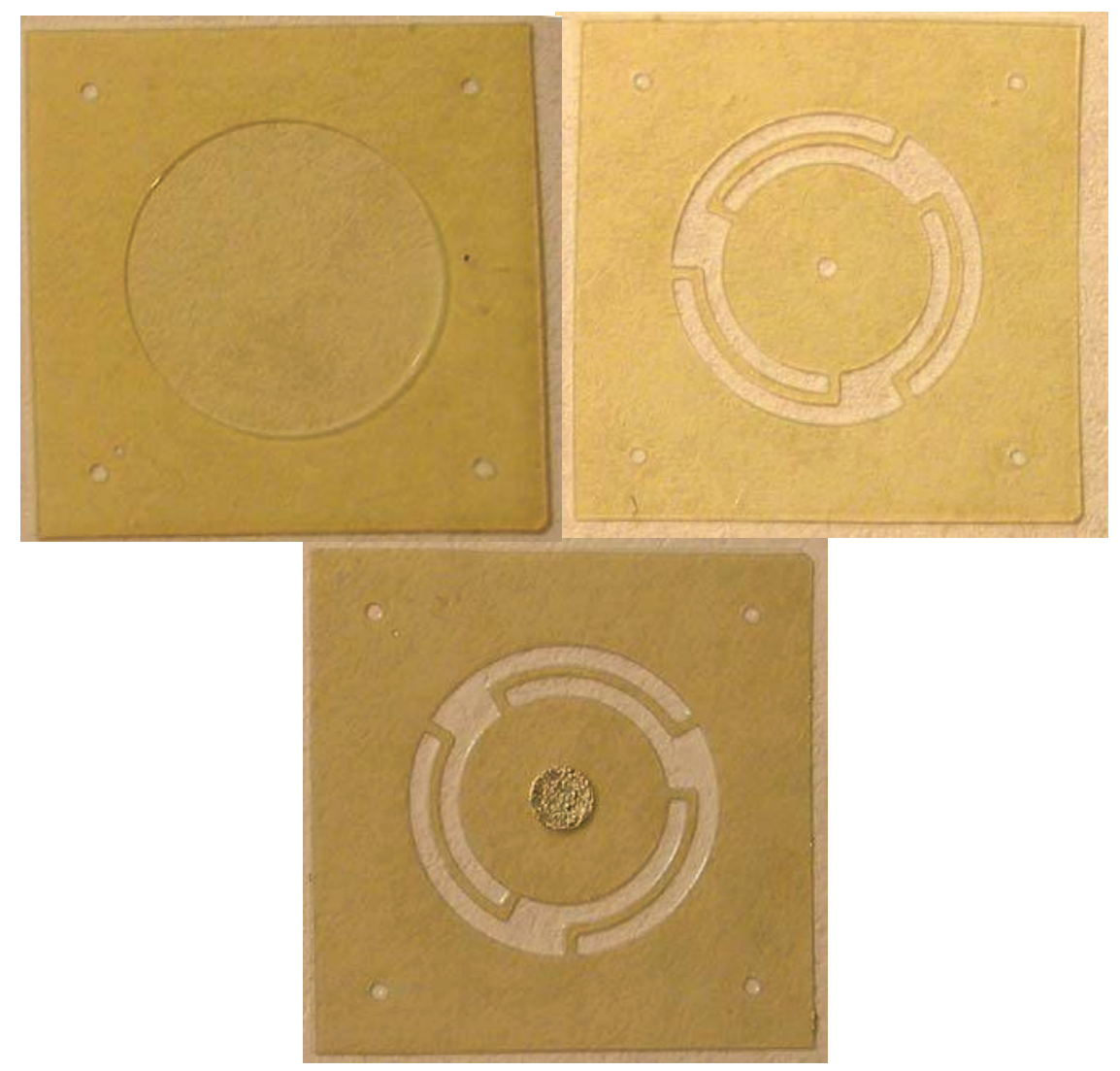

Figure 5. Elements of vibration sensor

1. Production of ferromagnetic materials with nanometer scale based on complex magnetically hard ferrites, ferroelectrics that will enable drastic energy savings in the account, because magnetization by the ferroelectric polarization of the material. Of greatest interest is manifested in the creation of template-ordered structures based on quantum-well materials (composites, and individual compounds) having ferroelectric and ferromagnetic properties. Expected use of chemical and electrochemical methods to obtain independent quantum dots, points of core - shell and their combinations on the basis of these materials.

2. Development of a obtaining method of the template - ordered porous alumina and the composite on its basis with antiferromagnets, since it is assumed that superparamagnetism in nanostructured magnetic materials can be controlled by contact with an antiferromagnet. The distance between the nanodots in the resulting structure may be $6-25 \mathrm{~nm}$, and the density of magnetic bits on the surface to reach $10^{12}$ dots per square inch. You can reach densities $20 \mathrm{Tbit} / \mathrm{inch}^{2}$. Such a structure could serve as a basis for magnetic-electric RAM (in the presence of electrodes recording - read), and devices such as HDD with perpendicular recording.

3. Development of methods and technologies introduction of magnetic particles in the template and the suppression of superparamagnetism by forming nanorods and item 2. Structures can be both homogeneous and sandwich, which will expand the number of magnetic states of a quantum rod, and a sharp increase the recording density.

4. Production of the piezogenerators for independent sources on the basis of nanosticks of the piezoelectrics, formed in the oxide-oriented array of nanotubes. Nanostructured piezoelectrics have a small life, which can be avoided by forming them into a matrix. As piezoelectrics can be used $\mathrm{ZnO}, \mathrm{ZnS}$, $\mathrm{BaTiO}_{3}$, and others even Si). As a complement possible visualization of mechanical stress through an LED system, formed in the same nanotube. 


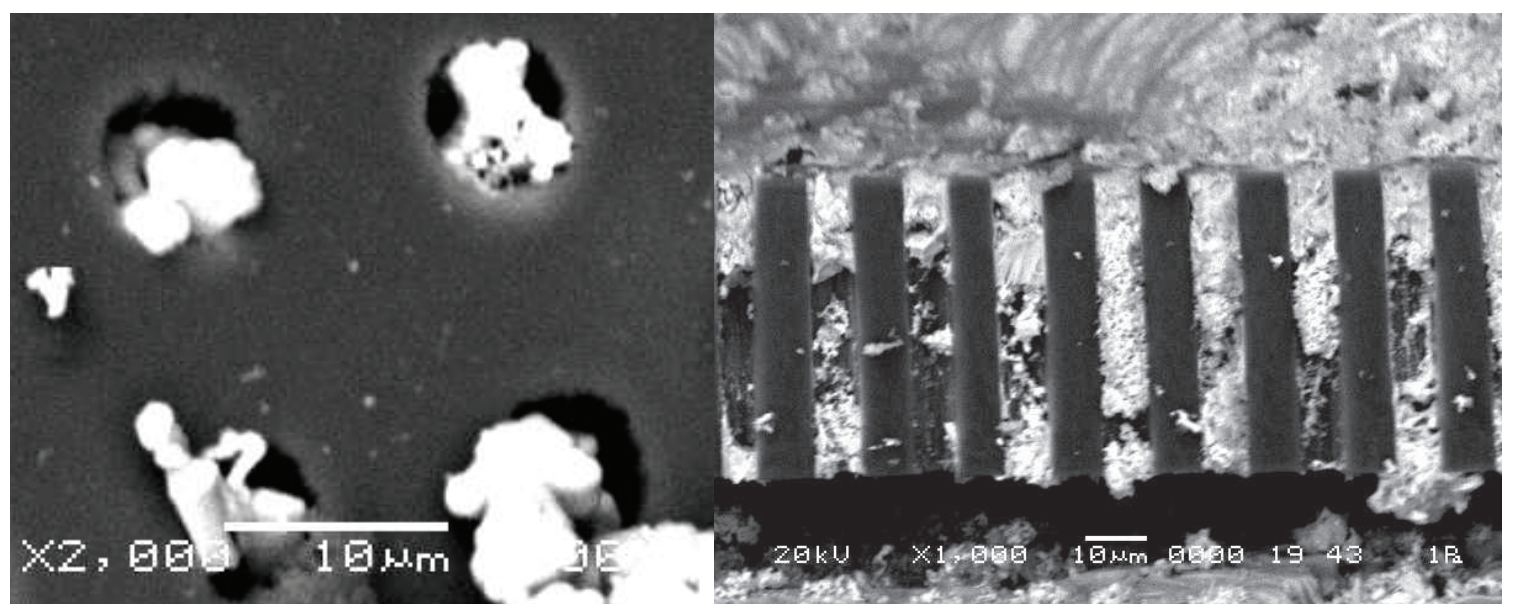

Figure 6. CdS in pores of $\mathrm{Al}_{2} \mathrm{O}_{3}$

Thus, the technology using aluminum oxide is a versatile tool for production of wide range of microdevices and nanoelements for various applications.

\section{References}

1. I.L. Grigorishin, Physico-technologic base of creation of hybrid IC for extremely conditions, Dr. Thesis, Minsk, 1988, p.34 (in Russian).

2. I.L. Grigorishin, G.I. Efremov, N.I. Mukhurov, P.E. Protas, Microcommutation devices on electrostatic control principle, Vide, couches, 1994, V.50, №271, P. 308-311. Russian)

3. I.L. Grigorishin et al, Ozone sensor based on thin film of NiO, Sensor, 2004, Vol.4, P. 4-10 (in 